\title{
Role of oxidatively-induced DNA damage and inflammation in radiation long term effects and carcinogenesis
}

\author{
Alexandros G. Georgakilas \\ Physics Department, School of Applied Mathematics and Physical Sciences, National Technical University of Athens \\ (NTUA), Zografou Campus, Athens, Greece
}

Correspondence: Alexandros G. Georgakilas. Address: Physics Department, School of Applied Mathematics and Physical Sciences, National Technical University of Athens (NTUA), Zografou Campus, Athens 15780, Greece.

Email: alexg@mail.ntua.gr

Received: October 16, 2013

Accepted: October 16, 2013

Online Published: October 17, 2013

DOI : 10.5430/jst.v3n6p1

URL: http://dx.doi.org/10.5430/jst.v3n6p1

\begin{abstract}
Ionizing radiation is undoubtedly the most frequently used means of cancer treatment especially in the case of solid tumors like breast, prostate etc. Significant evidence suggests that long-term effects of radiation in patients who have undergone even successful treatment include high oxidative stress and inflammation in their blood and various tissues and the underestimation of the so called "non-targeted" effects. This chronic stress can lead in some cases to malignant transformation (secondary primary cancers) after radiation treatment of primary tumors several years after the initial irradiation. This mini-review discusses the evidence for the role(s) of oxidative DNA damage and inflammation as one of the primary contributing factors in chronic tissue injury and secondary carcinogenesis. Several important questions arise like for example if based on this idea we can develop in vivo reliable predictive biomarkers for radiation-induced chronic injury and to pinpoint carcinogenesis precursor sites after radiotherapy treatment even years after the patient exposure.
\end{abstract}

\section{Key words}

Oxidative stress, DNA damage, Inflammation, Radiation therapy, Long-term effects

\section{I ntroduction}

A percentage of $60 \%-70 \%$ of cancer patients has received radiation treatment as a part of their therapeutic regime. The induction of chronic oxidative stress, DNA damage and inflammation are accepted as possible major contributors of tissue injury and possibly malignant transformation and initiation of secondary primary cancers in radiotherapy-treated patients ${ }^{[1]}$. Exposure to radiotherapy with total doses varying from $20-70 \mathrm{~Gy}$ is expected to produce a significant amount of clustered (complex) DNA lesions induced directly (acute effect) from radiation or indirectly through generation of chronic inflammation and oxidative stress in patients. Clustered DNA damage i.e. a dense synthesis of DNA lesions are poorly repaired and expected to produce mutagenic effects and pro-malignant sites through chromosomal instability in cells and tissues (cancer precursor sites). Therefore, there is a critical need to delineate the molecular mechanisms behind the processing (repair) of radiation-induced clustered DNA damage and radiation effects ${ }^{[2]}$. In addition, it is of high importance to develop reliable radiation biomarkers that will predict the acute and/or delayed injury to specific organs and 
tissues after radiation exposure (radiation therapy) and methodologies to assay those biomarkers, to facilitate precise and timely medical interventions and optimization of radiation therapy ${ }^{[3]}$.

Therefore our main hypotheses of this mini-review are: (1) that clustered DNA damage is an in vivo characteristic signature of radiation and the severity and complexity of clustered DNA damage can be used as a marker for biological response and cancer precursor sites induction (DNA damage, epigenetic changes and chromosomal breaks) and (2) that mitigation/amelioration of these end product effects (cancer precursors) can be achieved by use of dietary anti-oxidants and anti-inflammatory drugs underlying the central role of oxidative stress and inflammation in these long-term effects of radiation.

The critical target of ionizing radiation (IR) has traditionally been believed to be the DNA with relatively little substantial cellular effects from action on other molecules or especially outside the area of radiation. Most of traditional radiobiology has almost exclusively targeted this direct action of radiation. Relatively recently, other types of impact from IR have been detected i.e., that IR produces significant effects in "non-targeted" cells/tissues not directly interacting with radiation or radiation genotoxic products such reactive oxygen species (ROS). These short or even long-term effects include (but not limited to): increased DNA damage, inflammation, DNA repair gene and protein expression changes and genomic instability. DNA damage detected by a variety of techniques includes simple and complex oxidative DNA lesions and double-strand breaks (DSBs) ${ }^{[4]}$. These non-targeted effects have been investigated under a variety of conditions (for a recent review, please see $\left.{ }^{[5]}\right)$. The consequential non-targeted effects for specific circumstances have been designated as 'bystander effects, abscopal effects' cohort effects and adaptive effects ${ }^{[6]}$. Although the mechanisms behind this phenomenon still in principle remain under intensive investigation the involvement of oxidative stress, pro-inflammatory and inflammatory responses is considered fundamental ${ }^{[7]}$.

\section{Evidence on the role(s) of oxidative stress and inflammation}

An intensive research ofthe literature and always in relation to radiation-induced health risks for the human population and especially radiotherapy patients, underlines the necessity of targeting the mechanisms towards understanding the induction of oxidative stress and inflammation in radiation-induced injury in mammalian tissues and organs through targeted and non-targeted effects. The risk of radiation-induced late normal tissue injury can limit significantly the dose of radiation that can be delivered safely and efficiently to cancer patients with solid tumors. A variety of side effects and different types and levels of tissue injury manifested as tissue dysfunction and failure (associated with atrophy, fibrosis and/or necrosis) as well as vascular injury, have been reported in late responding normal tissue. These tissues usually are the central nervous system, gut, kidneys, liver, lungs, and skin. The exact mechanisms underlying the pathogenesis of radiation-induced long term effects have not been entirely and meticulously investigated. It has been proposed that the radiation-induced late effects are initiated, in part, by inflammation andchronic oxidative stress leading to DNA damage and potentially chromosomal instability ${ }^{[8]}$. Increased production of reactive oxygen and nitrogen species (ROS/RNS), which usually leads to lipid peroxidation, oxidation injury of proteins and DNA, as well as release of pro-inflammatory and/or various clastogenic (chromosome breaking) factors (tumor necrosis factor, free radicals, lipid peroxidation byproducts, inosine triphosphate and others) has been observed in vitro and in vivo. In the past ten years, evidence has accumulated for a novel biological phenomenon termed as "radiation-induced bystander effect" (RIBE) (discussed also above), wherein directly irradiated cells transmit "damaging" signals like soluble plasma clastogenic factors to non-irradiated cells and neighboring tissues thereby inducing a DNA damage response very similar to that of irradiated cells ${ }^{[9]}$. The in vivo though confirmation of the 'bystander' phenomenon is needed in order to eliminate uncertainties in the field of radiation therapy and biology and to provide a solid foundation for assessing the possible implications of this effect in humans exposed to ionizing radiations. Nuclear factor-kappa B (NF-kB) is a well known pivotal transcription factor for many signalling genes, including the inflammatory moleculecyclooxygenase-2(COX-2). It is expected that $\mathrm{NF}-\kappa \mathrm{B}$ plays an important role in the 'bystander' response primarily through COX-2 upregulation ${ }^{[10]}$. Future research projects will have to link for the first time all three poles of this complex phenomenon in vivo: the radiation-induced 
biological response (DNA damage, epigenetic modifications, apoptosis/senescence and chromosomal breaks) with oxidative stress (ROS, lipid peroxidation, antioxidant enzymes) and inflammation (release of cytokines, macrophages) pathways. Therefore, the suggested interdisciplinary studies are considered a significant advancement in the field with applications ranging from radiotherapy to human population health.

\section{Conclusions and future steps}

The proposed work for the near future has to utilize systematic and innovative carefully designed in vivo experiments to address important questions related to the induction, processing and biological significance (cancer risk) of radiation. In addition, it has been nearly impossible to find a single biological metric for estimating the short- and long-term effects of ionizing radiation especially in the non-irradiated areas (bystander). In order to address all these issues, we have to initiate a cohesive and integrated multidisciplinary effort. The first, innovative aspect of the near-future research, it has to be the determination of cluster damage levels in vivo (human patients and animals) and the bilateral targeting of two contributing factors: oxidative stress and inflammation (mouse and human models). The second innovative aspect, should be the analytical study of the role(s) and mechanisms of non-targeted effects in vivo combined with the cluster processing, inflammation, epigenetic changes and chromosomal aberrations which are expected to be strongly associated. Finally, the short- and long-term design of appropriate studies has to include another innovative element i.e. the realistic simulation of the patient's response to radiation therapy. To our knowledge, these hypothetical studies will be the only ones so far that will examine such a variety of radiation response mechanisms at the mammalian organism level (mice strains and patients). Using mice with or without antioxidant dietary/anti-inflammatory supplementation will offer unique and applicable to human health, mechanistic insights for the modulation and mitigation of cancer risk due to exposure of population to environmental radiation or radiotherapy via elimination of the so called 'cancer precursors' like clustered DNA damage, epigenetic changes, chromosomal aberrations and modulation of the bystander effects etc. This new hypothesis and unconventional combined methodologies are expected to lead to a major breakthrough by showing clear evidence for a unifying model between irradiated tissue and tumor development with an impact beyond the specific research domain of radiobiology and distinctive applications in a variety of environmental and medical conditions and interventions affecting human health.

\section{Acknowledgement}

The author acknowledges the funding support by an EU grant MC-CIG-303514, THALIS grant (Proposal no: 356 MPN) of the Greek General Secretariat of Research and Technology (GSRT) and COST Action CM1201 "Biomimetic Radical Chemistry". I apologize to all investigators whose meritorious work could not be cited in this article due to space limitations.

\section{References}

[1] Nowsheen S, Aziz K, KrystonTB, Ferguson NF, Georgakilas AG. The interplay between inflammation and oxidative stress in carcinogenesis. CurrMol Med. 2012; 12:672-680.PMid:22292435http://dx.doi.org/10.2174/156652412800792642

[2] Stewart RD, Yu VK, Georgakilas AG, Koumenis C, Park JH, Carlson DJ. Effects of radiation quality and oxygen on clustered DNA lesions and cell death. Radiat Res. 2011;175:587-602.PMid:21823972http://dx.doi.org/10.1667/RR2663.1

[3] Werbrouck J, Ost P, Fonteyne V, De Meerleer G, De Neve W, Bogaert E, BeelsL, Bacher K, Vral A, Thierens H. Early biomarkers related to secondary primary cancer risk in radiotherapy treated prostate cancer patients: IMRT versus IMAT. RadiotherOncol. 2013;107:377-381.PMid:23791364http://dx.doi.org/10.1016/j.radonc.2013.05.014

[4] Dieriks B, De Vos W, Baatout S, Van Oostveldt P. Repeated exposure of human fibroblasts to ionizing radiation reveals an adaptive response that is not mediated by interleukin- 6 or TGF- $\beta$. Mutat Res.

2011;715:19-24.PMid:21784085http://dx.doi.org/10.1016/j.mrfmmm.2011.07.002 
[5] Sprung CN, Ivashkevich A, Forrester HB, Redon CE, Georgakilas AG, Martin OA. Oxidative DNA damage caused by inflammation may link to stress-induced non-targeted effects. Cancer Lett. 2013.PMid:24041866http://dx.doi.org/10.1016/j.canlet.2013.09.008

[6] Blyth BJ, Azzam EI, Howell RW, Ormsby RJ, Staudacher AH, Sykes PJ. An adoptive transfer method to detect low-dose radiation-induced bystander effects In vivo. Radiat Res. 2010;173:125-137.PMid:20095844http://dx.doi.org/10.1667/RR1899.1

[7] Kryston TB, Georgiev A, GeorgakilasAG.Role of oxidative stress and DNA damage in human carcinogenesis. Mutat Res. 2011; 711: 193-201.

[8] Zhao W, Robbins ME: Inflammation and chronic oxidative stress in radiation-induced late normal tissue injury: therapeutic implications. Curr Med Chem. 2009;16:130-143.PMid:19149566http://dx.doi.org/10.2174/092986709787002790

[9] Prise KM, O'Sullivan JM: Radiation-induced bystander signalling in cancer therapy. Nat Rev Cancer. 2009; 9: 351-360. PMid:19377507 http://dx.doi.org/10.1038/nrc2603

[10] Hei TK, Zhou H, Ivanov VN, Hong M, Lieberman HB, Brenner DJ, Amundson SA, Geard CR: Mechanism of radiation-induced bystander effects: a unifying model. J Pharm Pharmacol.2008;60:943-950.PMid:18644187

http://dx.doi.org/10.1211/jpp.60.8.0001 